\title{
Information, Entropy Decrease and Simulations of Astrophysical Evolutions
}

\author{
Chang Yi-Fang* \\ Department of Physics, Yunnan University, China
}

*Corresponding author: Chang Yi-Fang, Department of Physics, Yunnan University, Kunming 650091, China, Email: yifangch@sina.com

\section{Research Article}

Volume 5 Issue 2

Received Date: June 23, 2021

Published Date: July 21, 2021

DOI: $10.23880 / p s b j-16000181$

\section{Abstract}

Entropy is a great development in science. We proposed that entropy decrease due to internal interactions in the isolated system is possible. We define the entangled scale, which mainly involves the number $n$ and entangled degree. Since coherence, entanglement and correlation are all internal interactions in information systems, we discuss quantitatively entropy decrease along coherence, and entropy increase only for incoherence. From beginning quantum heat engine, we must systematically study quantum thermodynamics. Based on some astrophysical simulation models, they shown that the universe evolves from disorder to structures, which correspond to entropy decrease. This is consistence with theoretical result. The simulation must be an isolated system only using internal gravitational interactions.

Keywords: Entropy; Information; Coherence; Astrophysics; Evolution; Simulation

\section{Introduction}

Great scientist Eddington said: "The law that entropy always increases holds, I think, the supreme position among the laws of Nature" [1]. So far, entropy as a great development in science points out that it is always increase and tend to disorder in isolated system. Its any violation seems need Maxwell demon [2,3]. We proposed that if interactions, fluctuations and their magnified exist among various subsystems of an isolated system, entropy decrease in the isolated system is possible [4,5], which includes physics [6-9], chemistry [10,11], biology [12-14], astronomy $[15,16]$ and social sciences [17-19]. For attractive process, internal energy, system entropy, and nonlinear interactions, etc., an isolated system may form a self-organized structure with lower entropy. Some possible entropy decreases are calculated quantitatively [6,9]. It agrees probably with the first basic theorem proposed by Clarke AC (1917-2008).
Recently, we emphasized that preconditions of entropy increase are 1) for isolated systems; 2) various internal interactions in system must be neglected; 3) they must be thermal equilibrium processes. We proposed possible entropy decrease due to fluctuation magnified and internal interactions (these are namely Maxwell demon) in isolated systems, and research various possible entropy decreases in physics, which include phase transformation from disorder uniformity to order state. The solidification forms spontaneously an order structure, and it may be process of entropy decrease. We proposed that entropy decrease exists necessarily in self-assembly as isolated system, and discussed the molecular motor and entropy decreases in biology. We proposed quantitatively a total formula of entropy change for universal evolution of any natural and social systems. As long as we break through the bondage of the second law of thermodynamics, the rich and complex world is full of examples of entropy decrease [20]. In this paper, we discuss 
possibly entropy decrease for entanglement, coherence in systems, and for some astrophysical simulation models.

\section{Information and Entanglement}

Classical information theory studies non-interference information in the same channel, i.e., information is independent. This corresponds to that base of entropy is the independence of all subsystem, i.e., additive entropy. Wang, et al. researched the experimental tests for the second law of thermodynamics $[21,22]$. It is very meaning.

A popular definition of information is the negative entropy. In an isolated system information will decrease, which corresponds to the second law of thermodynamics. A certain population can be used as an isolated system. If they communicate only, their information will be decreased; but, if they discuss, argue and interaction each other, many new ideas may be generated, and information will increase [1719]. Such the democratic and equal society is more conducive to the development of science and the social progress.

DNA replication, cell reproduction, etc. are all increasing information through internal interactions. Although the system must input energy, etc., in a longer time period, but the system can be considered isolated for a short time period. One of the characteristics in biology is that the gene replications are passed on from generation to generation, and information is repeated and not decreased. Even internal mutations increase information, and constantly increasing biological complexity.

Now the quantum entangled state is approved by some experiments [23-25]. A classical example is the Which Way (WW) experiments by double-path interferometer and atom interferometer, etc [26-29]. It is difficult that determinant electron, neutron, photon pass through which way. This implies the complementarity (the mutually exclusive nature of the wave and particle concepts) [30].

Monroe, et al. completed the superposition and entanglement of Beryllium [31]. Polzik, et al. proposed the method for entanglement of two quantum states [32]. Further, it will be that hundreds of millions of atoms entangled. Quantum entanglement of macroscopic objects contains entangled spin of Cs gas about $10^{12}$ atoms for $0.5 \mathrm{~ms}$. If it is fermion, it will violate Pauli Exclusion Principle (PEP) [7,33-37], and short time corresponds to high energy; if it is boson, it will correspond to BEC at high temperature. This can be related to macromolecular formation, atomic groups, chemistry, biological composition, etc., until the unity of man and nature. In short, we need to be studied in depth many basic problems in particle physics.
The quantum entangled state possesses some characters, for example, coherency, nonlocality, quantum teleportation and superluminal, we think that it should be a new fifth interaction.

Based on the classification of the timelike and spacelike intervals, one does not add any hypothesis, we necessarily derived the two symmetrical types of topologically separated structures: the subliminal $\mathrm{v}<\mathrm{c}$ Lorentz transformation (LT) and the superluminal $\bar{v}>\mathrm{c}$ general Lorentz transformation (GLT) $[38,39]$. Wang, et al. demonstrated the gain-assisted superluminal light propagation [40]. Mugnai, et al. observed superluminal behavior in wave propagation $[41,42]$. The quantum entangled states have different phases. These phase velocities may be superluminal, i.e., "spooky action at a distance" (Einstein), and may probably apply GLT $[40,41]$. GLT shows the nonlocality between phases.

It is known that the partial entropy of entanglement is:

$$
E_{p}\left(\mid \psi>_{A B}\right)=S\left(\rho_{A}\right)=-t r^{(A)}\left(\rho_{A} \log \rho_{A}\right) .
$$

Here $S\left(\rho_{A}\right)$ is von Neumann entropy [43].

We should investigate entropy and information in entanglement, and propose the entangled scale $s_{e t l}(n, d)$ , which mainly involves the number $(n>1)$ of entangled particles or systems, and entangled degree $(d=1,2,3 \ldots)$. In two dimensional space, it is:

$$
s_{e t l}(n, d)=n \vec{i}+d \vec{j}
$$

If this scale changes, its rate will be

$$
r_{e t l}(n, d)=\frac{d s_{e t l}}{d t}
$$

Moreover, there are also $\frac{\partial s_{e t l}}{\partial n}$ and $\frac{\partial s_{e t l}}{\partial d}$. The larger the number, the larger the general spatial scale, the entanglement is less easy.

Similar, we may definite the coherence scale $S_{c h r}$ and so on. Further, we should discuss the corresponding entropy and information.

Moreover, there are also uncertainties about information, such as conservation of information is inconsistent with the hairrous law of black hole.

\section{Quantum Theory and Entropy Decrease}

In quantum statistics the free energy corrected to Maxwell-Boltzmann (MB) value is [44]: 


$$
F=F_{M B} \pm \frac{\pi^{3 / 2}}{2 g} \frac{N^{2} \hbar^{3}}{V T^{1 / 2} m^{3 / 2}}
$$

where and later the upper sign corresponds to Fermi-Dirac (FD) statistics and the lower sign corresponds to BoseEinstein (BE) statistics. Correspondingly, the entropy is:

$$
S=-\left(\frac{\partial F}{\partial T}\right)_{V}=S_{M B} \pm \frac{\pi^{3 / 2}}{2 g} \frac{N^{2} \hbar^{3}}{V T^{3 / 2} m^{3 / 2}}
$$

Therefore, $d S=S-S_{M B}>0$ for Fermi gases, and $\mathrm{d} S<0$ for

Bose gases, i.e., the entropy in BE statistics is smaller than in MB statistics. In FD statistics the quantum exchange effects lead to the occurrence of an additional effective repulsion between the particles, and in BE statistics there is an effective attraction between the particles [44]. This is consistent with entropy decrease for attractive process $[4,5]$.

The second virial coefficient may be calculated with allowance for the quantization of the binary interaction of the gas particles, and the atoms obey BE statistics. The free energy is [44]:

$$
F=F_{i d}-\frac{8 N^{2}}{V}\left(\frac{\pi \hbar^{2}}{m}\right)^{3 / 2} Z_{\text {int }} T^{-1 / 2} .
$$

Correspondingly, the entropy is:

$$
d S=S-S_{i d}=-\frac{4 N^{2}}{V}\left(\frac{\pi \hbar^{2}}{m}\right)^{3 / 2} Z_{\text {int }} T^{-3 / 2} .(7)
$$

This is smaller than entropy of ideal gases [7].

It is known that entropy is measurement of disorder in a system [45]. In phase transformation the crystallization of an over-cooling liquid or of a supersaturated solution is surely an ordering process. The cooling principle is entropy reduced. The difference of entropies between the normal state and the superconductive state is:

$$
d S=S_{s}-S_{n}=\frac{H_{c} V}{4 \pi} \frac{\partial H_{c}}{\partial T}
$$

where $S_{s}$ is entropy of the normal state, and $S_{s}$ is one of the superconductive state. Such $\mathrm{dS}<0$ for $\frac{\partial H_{c}}{\partial T}<0$. A superconducting state is more order than a normal state. This phase transformation from the normal state to the superconducting state is a condensation process. Generally, any condensation process, in which attractive interactions exist, should be one of entropy decrease.

In the Ginzburg-Landau theory the difference in the free energies of the superconducting and normal states:

$$
F_{s}-F_{n}=-V\left(a^{2} / 2 B\right)\left(T_{c}-T\right)^{2} .
$$

Correspondingly, the entropy is:

$$
d S=S_{s}-S_{n}=-V\left(a^{2} / B\right)\left(T_{c}-T\right) .
$$

Such $\mathrm{d} S<0$ for $\mathrm{T}<T_{c}$.

The occurrence of superfluidity in a Fermi system is due to the Cooper effect, the formation of bound states (pairing) by mutually attracting particles. We predict that entropy will decrease for the Cooper effect, further, for BEC, for superconductivity and superfluidity. The superfluid helium and its fountain effect must suppose that the helium does not carry entropy, so that the second law of thermodynamics is not violated [45]. It shows that superfluidity will possess zero-entropy, but this cannot hold because zero-entropy corresponds to absolute zero according to the third law of thermodynamics. For the liquid or solid $\mathrm{He}^{3}$ the entropy difference [45] is $\Delta S=S_{l}-S_{s}>0$ (for higher temperature),

$=0$ (for $\mathrm{T}=0.3 \mathrm{~K}$ ), $<0$ (for lower temperature). Such a solid state with higher entropy should be disorder than a liquid state in lower temperature. Of course, the above examples are not already under the thermodynamic equilibrium condition. In the evolutionary process and the phase transformation, the systems cannot be in thermal equilibrium states.

When there is a critical point, a continuous transformation can be effected between any two states of the substance [44]. Interactions between the molecules are different for the two states, and internal symmetries are different, so entropies are also different. In the unsymmetrical phase, the entropy is [44]:

$$
S=S_{0}+\left(a^{2} / 2 B\right)\left(T-T_{c}\right)
$$

At the transformation point itself, this expression becomes $S_{0}$. For $\mathrm{T}>T_{c}, d S=S-S_{0}>0$; but for $\mathrm{T}<T_{c}, d S=S-S_{0}<0$. A symmetrical state is more order than unsymmetrical state. At a critical point of phase transformation of the second kind, there is [7]:

$$
\left.d S=S-S_{0}=-a \eta^{2}<0 \text { (for } a>0\right),(12
$$

where $\eta$ is an order parameter. These results are similar in the Landau-Devonshire theory and the Landau-de Gennes theory of phase transformation. 
In a system with internal interactions, the fluctuation can be magnified, for example, in those processes of phase transformation. When the order parameter of a system comes to a threshold value, a phase transformation occurs, and selforganization will take place. Simultaneously, the entropy will decrease continuously, and a final state with lower entropy will be reached. In this case various microscopic states are not equally probability. If entropy is seen as a degree of freedom, the interaction will reduce the degree of freedom.

In phase transformation, structures and symmetries are different, corresponding internal energies and entropies are also different. At a critical point of phase transformation, entropy can increase or decrease, i.e., possesses two-direction property. This corresponds to reversibility of transformation between order and disorder. Of course, the general phase transformation is an open system. But, if input energy is interrupted at the critical point, it will become an isolated system. Although in an isolated system the gravitational field cannot be screened completely, but the electromagnetic field may be screened completely. It is very easily that strong and weak interactions as short-distance ones are screened.

The specific heat $C_{V}=T d S / T$ is anomalous, i.e., entropy is anomalous. This implies entropy decrease [4-20]. A nucleation in phase transformation for solids, which may be formed due to thermal fluctuation, is an ordered process. The second law of thermodynamics is corrected, it will be conducive to develop freely theories on nucleation and grow in dynamics of phase transformations.

$$
S=\int_{0}^{T}\left(C_{V} / T\right) d T
$$

The second law of thermodynamics is based on an isolated system and statistical independence [45]. If fluctuations magnified due to internal interactions exist in the system, entropy will decrease possibly [4-20].

\section{Coherence and Entropy Decrease}

In quantum information there are correlation, entanglement and coherence $[46,47]$, which are generally all internal interactions in systems, and not only for quantum.

Let $\{|0>| 1>$,$\} as a set of bases in a two-dimensional$ Hilbert space, whose density matrix is:

$$
\rho=\left(\begin{array}{cc}
\rho_{00} & \rho_{01} \\
\rho_{10} & \rho_{11}
\end{array}\right)
$$

Here the principal diagonal $\rho_{00}, \rho_{11}$ are two nonnegative real

numbers, which represent the probability of the ground state $\mid 0>$ and $\mid 1>$, respectively; the secondary diagonal $\rho_{01}, \rho_{10}$ are two complex numbers, which represent the quantum coherence, and if they are 0 , the system will have not the quantum coherence.

For the density ensemble, entropy of the ensemble is defined as:

$$
S=-k \operatorname{Tr}(\rho \ln \rho) \cdot(15)
$$

For the diagonal density

$$
S=-k \sum_{n} \rho_{n n}^{(\text {diag })} \ln \rho_{m n}^{(\text {diag })} .
$$

For a pure ensemble

$$
\rho=\left(\begin{array}{ccccc}
0 & \ldots & 0 & \ldots & 0 \\
\ldots & \ldots & \ldots & \ldots & \ldots \\
0 & \ldots & 1 & \ldots & 0 \\
\ldots & \ldots & \ldots & \ldots & \ldots \\
0 & \ldots & 0 & \ldots & 0
\end{array}\right) \cdot(17)
$$

$\mathrm{S}=0$ is the maximum order.

For a completely chaotic mixed ensemble

$$
\rho=\frac{1}{N}\left(\begin{array}{ccccc}
1 & \ldots & 0 & \ldots & 0 \\
\ldots & \ldots & \ldots & \ldots & \ldots \\
0 & \ldots & 1 & \ldots & 0 \\
\ldots & \ldots & \ldots & \ldots & \ldots \\
0 & \ldots & 0 & \ldots & 1
\end{array}\right) \cdot(18)
$$

$\mathrm{S}=\mathrm{k} \ln N$ is the equally probability and the statistics independent, and $\mathrm{S}$ is the maximum.

From complete chaos to order, entropy decreases. It corresponds to the non-diagonal density, and has coherent, and is partially ordered and entropy decrease.

For Eq.(14) $|\rho|=\rho_{00} \rho_{11}-\rho_{10} \rho_{01}$. For the diagonal matrix $|\rho|=\rho_{00} \rho_{11}$ is the maximum, and density always greater than $0,|\rho|$ becomes smaller. Interference reduces entropy decrease.

Assume that $\{i>\}_{i=1}^{d}$ are the given set of standard

orthogonal bases in the d-dimensional Hilbert space. If the density operators of the quantum system can be diagonalized, the quantum state will be incoherent, otherwise it is quantum coherent.

Quantum coherence and quantum entanglement represent two fundamental features of nonclassical systems that can each be characterized within an operational resource theory. Chitambar, et al. unified the resource theories of entanglement and coherence by studying their combined behavior in the operational setting of local incoherent operations and classical communication (LIOCC) [48]. 
The incoherent operation does not cause the coherence increase of the quantum state, that is, the incoherent operation may reduce the coherence of the quantum state on any quantum state. The incoherent and decoherence should correspond to the entropy increase without interaction. From this it may be extended to the general state.

Recently, coherence, incoherence and correlation, entanglement are discussed widely [49-68]. Let $\rho$ is a density operator of d-dimensional Hilbert space, such its relative entropy coherence and system entropy satisfy the following uncertain relation:

$$
C_{r}(\rho)+S(\rho) \leq \log _{2} d .(19)
$$

It is namely that the relative entropy coherence of any quantum state $\rho$ does not exceed the information function:

$$
C_{r}(\rho) \leq I_{t}(\rho) \cdot(20)
$$

This shows that the larger the entropy of the quantum system, the less coherence; conversely, the more coherence of the quantum system, the smaller the entropy of the quantum system. In a word, entropy decreases along coherence, and entropy increases only for incoherence. This is impossible that entropy always increases for coherence and incoherence. It is completely consistent with my theory [4-20].

The actuator is a material that can change its characteristics according to the changing system state [58]. Modern smart materials are sometimes called "life in the non-living world" [59]. This may even be related to animism. This might make Maxwell demon [2,3].

Cellular automata may aggregate automatically $[60,61]$, and perhaps produce results violating the entropy increase principle [4-20].

The internal interactions, and their fluctuation and amplifications can form structures and derive order, and can obtain entropy decrease and self-organization based on the nonequilibrium statistical physics. An example is Vicsek model $[66,67]$. Biological evolution is mainly selfinteractions [68].

Scully, et al. designed a photonic gas heater [69]. For this case, the second law of thermodynamics is violated on the surface, and the efficiency of heat engine may be greater than that of the Carnot cycle. Quan, et al., studied a new quantum heat engine (QHE), which is assisted by a Maxwell's demon. The QHE requires three steps: thermalization, quantum measurement, and quantum feedback controlled by the Maxwell demon. They derived the positive-work condition and operation efficiency of this composite QHE. The essential role of the demon is explicitly demonstrated in this macroscopic QHE [70]. If Maxwell demon exists in quantum cases, it will improve the efficiency of quantum heat engines. We must systematically study quantum thermodynamics.

Hackermuler, et al. discussed decoherence of matter waves by thermal emission of radiation [71]. Decoherence enhances along increasing temperature, namely decoherence is associated with temperature T. Entanglement should be the same result. In the human body and parapsychology, a high temperature is dizzy.

\section{Simulation of Evolutional Universe}

It is known that stars are formed from nebula through gravitational self-interactions. Now an important way of modern astronomy is the evolutional simulation, especially, for the evolution of universe [72]. So far, there are some wellknown astrophysical simulation models.

In 2005, the Max Planck Institute for Astrophysics completed Millennium Simulation Project [73-76]. This is the square box (about 2.3 billion light-years) with a side length of $500 / \mathrm{h}$, in which $\mathrm{h}$ is the Hubble constant. It contains 21603 dark matter particles, i.e., it is equivalent to simulating the evolution of a multi-body system composed of 10 billion particles. This created an image of a cosmic network, whose structure is very similar to some spatial distribution of galaxies (Figure 1).

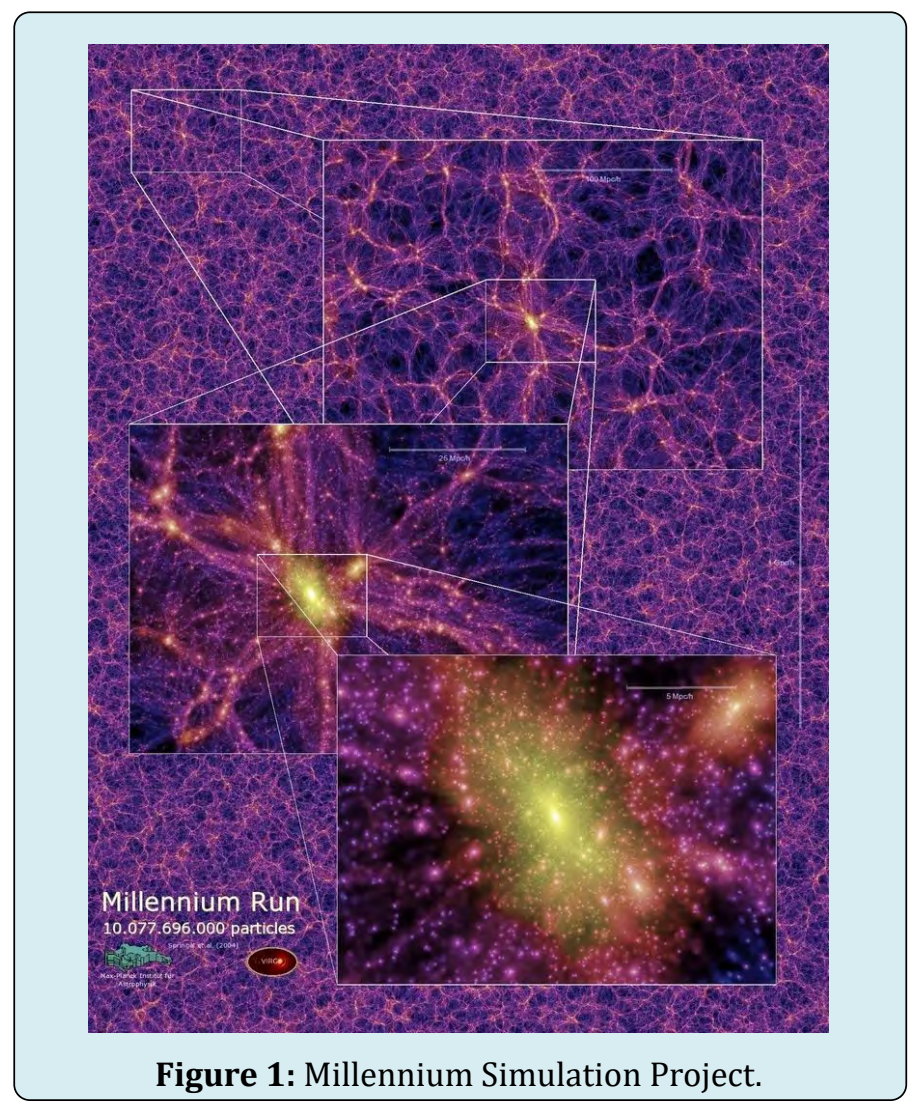


Further, in 2019 Millennium Simulation is expanded to 50403 to reproduce the formation and evolution of galaxies under the cold dark matter model, statistically compared with the galactic properties observed, and test current cosmological theories, and explain the results of SDSS, and try to answer fundamental astronomical questions on the evolution of universe, and the nature of dark matter and dark energy.

In 2009 China developed a "Phoenix" simulation for highprecision galaxy cluster. In 2010 Computational Cosmology Consortium of China (C4) launched a large cosmological numerical simulation program, "Pangu Project" (Figure 2).

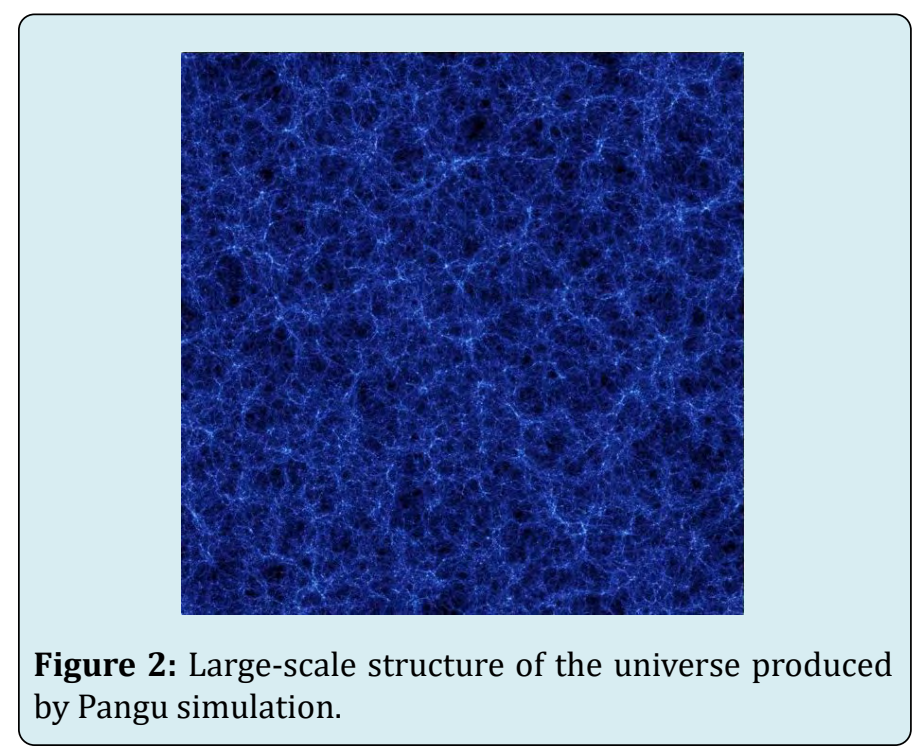

Other well-known cosmological simulations include FIRE simulation, ERIS simulation, Illustris simulation and next-generation Illustris simulations, MultiDark simulation, and the cluster of three hundred galaxies simulation, etc (Figure 3).

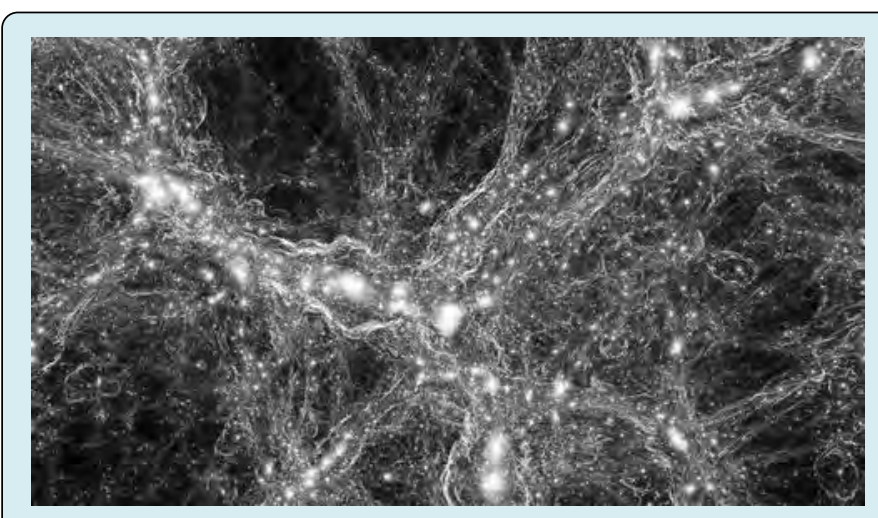

Figure 3: TNG Collaboration.

In 2012, astrophysicists used computers to simulate the most realistic evolution of the universe, which includes all celestial activity from the big bang to the present, spanning about 14 billion years. They condensed the whole process into a video about 78 seconds long by Arepo simulator (Figure 4).

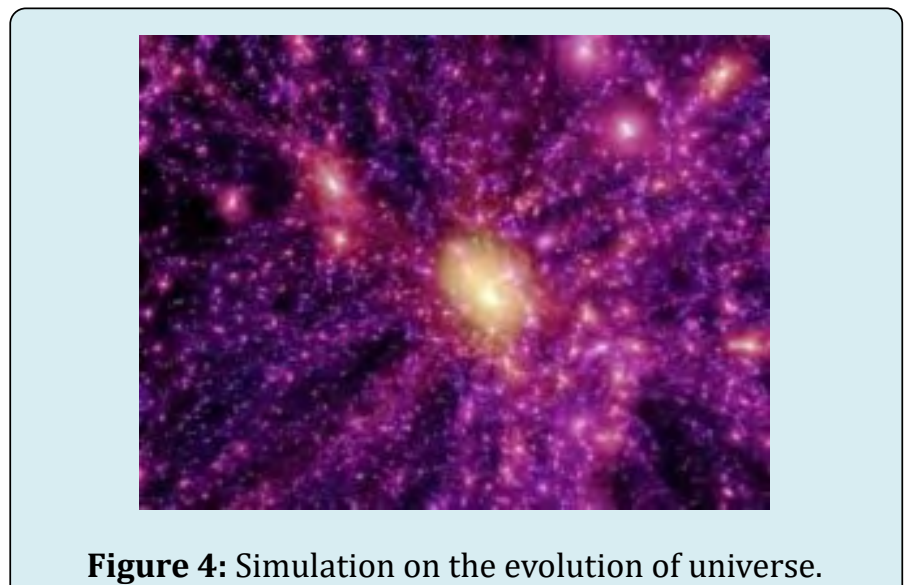

Vogelsberger, et al. proposed a model for cosmological simulations of galaxy formation physics [77]. They reported a simulation model of the Universe that follows the evolution of both visible and dark matter starting 12 million years after the Big Bang. This simulation covers a region of space big enough to be representative of the whole Universe, and traces 13 billion years of cosmic evolution with 12 billion resolution elements in a cube of 106.5 megaparsecs (350 million light years). It produces a mixture of galaxy shapes that fit observations well, and accurately recreates the largescale distribution of galaxy clusters and neutral gas in the Universe, as well as the hydrogen and heavy element content of galaxies [78]. Gibney reviewed this model universe recreates evolution of the cosmos [79], and this successful simulation lends weight to standard model of cosmology (Figure 5).

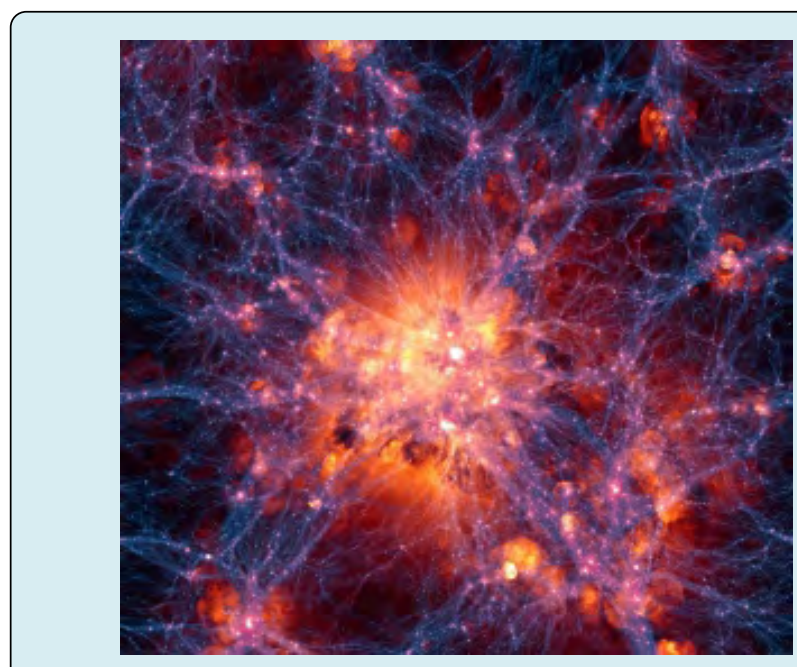

Figure 5: Simulation of large galaxy cluster. 
Brook, et al. studied galaxy formation using simulations [80].

In 2014, astronomers announced that after three months calculation used $8000 \mathrm{CPU}$ connected, a computer simulation of the evolution of the universe was born and evolved from dark matter (Figure 6).

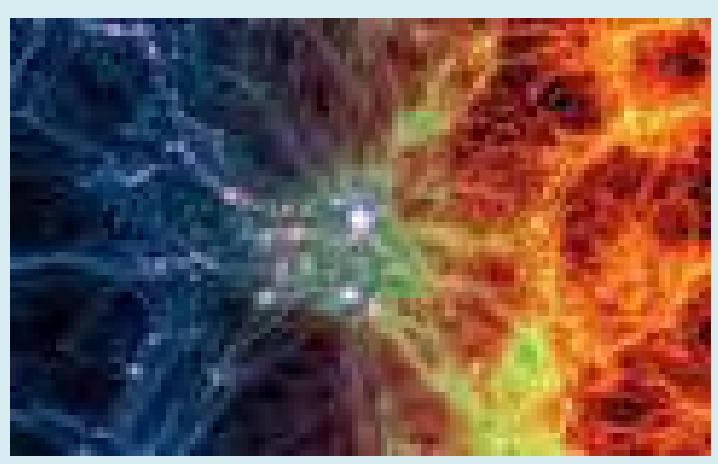

Figure 6: Simulation of the evolution of the universe.

On 12 October 2020, scientists have released a newly completed and state-of-the-art galaxy evolution simulation. TNG50 is the most detailed large-scale cosmic evolution simulation, which allowing researchers to study in detail how galaxies are formed (Figure 7).

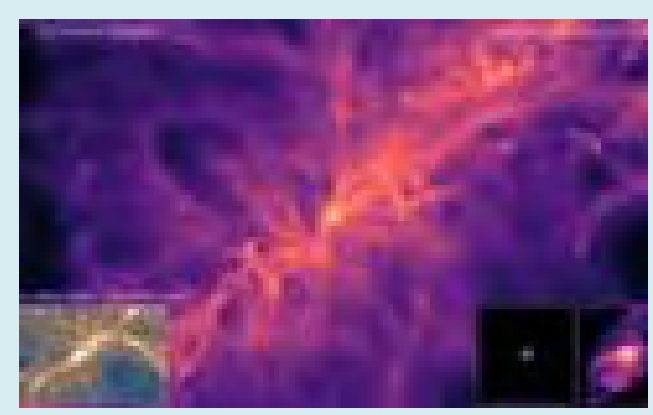

Figure 7: Large-scale cosmic evolution simulation.

These simulations agree well with observations of the evolutional Universe [81,82].

Moreover, Springel, et al. used cosmological smoothed particle hydrodynamics simulations as a hybrid multiphase model for star formation [83]. Saro, et al. discussed properties of the galaxy population in hydrodynamical simulations of clusters [84]. Libeskind, et al. researched satellite systems around galaxies in hydrodynamic simulations [85]. Sijacki, et al. proposed a unified model for AGN feedback in cosmological simulations of structure formation [86]. Dolag, et al. searched substructures in hydrodynamical cluster simulations [87]. Springel, et al. discussed the Galilean- invariant cosmological hydrodynamical simulations on a moving mesh [88]. Wiersma, et al. predicted the effect of variations in the input physics on the cosmic distribution of metals by simulations [89]. Genel, et al. discussed tracer particles in astrophysical fluid simulations [90]. Marinacci, et al. researched the formation of disc galaxies in highresolution moving-mesh cosmological simulations [91].

Since entropy is a measurement of disorder [45], some processes of the celestial evolutions must be order, and entropy decrease $[4,15]$. For instance, a disorder nebula becomes an order star due to the internal gravitational interaction in systems.

Almost all matter in interstellar space can be considered to be plasma, an ionized gas consisting of electrons, ions, and neutral atoms or molecules [92]. The entropy of plasma is

$$
S-S_{0}=3 k N_{\text {tot }} \ln \left[\left(\frac{T}{T_{0}}\right)\left(\frac{\rho_{0}}{\rho}\right)^{\gamma-1} \frac{1+\alpha}{1+\alpha_{0}}\right] .
$$

where $\gamma=c_{P} / c_{V}, \gamma \approx 4 / 3$ for the polyatomic gases. In an isolated system of plasma, the ions will attract electrons to neutral atoms. Since the plasma is often taken to be in local thermodynamic equilibrium [92], and the total mass of an isolated system is constant, temperature $\mathrm{T}$ and average density $\rho$ are invariant. Therefore, the final ionicity changes smaller, so that the final entropy decreases $[4,15]$.

The galaxy may be regarded as an isolated system [93]. General celestial bodies originate from nebula. For classically isolated gas, entropy is [45]:

$$
S(T, V)=S_{0}+n R \ln \left[\left(\frac{T}{T_{0}}\right)^{\alpha} \frac{V}{V_{0}}\right] .
$$

Therefore, entropy should decrease when the volume becomes smaller or the temperature decreases. Entropy of the ideal gases is:

$$
S=C_{V} \ln T+n R \ln V+S_{0}
$$

For an equal-temperature process $\mathrm{T}=$ constant,

$$
d S=S_{f}-S_{i}=n R \ln \left(V_{f} / V_{i}\right) .
$$

This decreases $\mathrm{dS}<0$, when $V_{f}<V_{i}$, i.e., for the attractive process. Conversely, the entropy increases $\mathrm{dS}>0$, when $V_{f}>V_{i}$. The entropy of non-ideal gases is [44]:

$$
S=S_{i d}+N \log (1-N b / V) .
$$

This is smaller than one of ideal gases, since $b$ is four times volume of atom, $b>0$. It corresponds to the existence of 
interaction of the gas molecules, and average forces between molecules are attractive [94].

According to the second law of thermodynamics, all systems in Nature will tend to "heat death" [94], But, world is not pessimistic always [4,15]. In some cases, internal interactions are very important. The gravitational interactions produce various ordered stable stars and celestial bodies. Various stable objects and their formations from particles to stars are accompanied with internal interactions, which have implied a possibility of entropy decrease.

When a black hole absorbs matter, its mass and entropy increase. Conversely, if a black hole evaporates matter [95], so its mass and entropy should decrease. Both are all open systems. Such thermodynamics of black hole should be the theory of dissipation structure, whose entropy decreases possibly. Further, this is impossible that both contrary collection and evaporation of black hole are all entropy increase. For opposite black hole and white hole, one is entropy increase, so another must be entropy decrease [16].

Various computer simulations show that the universe evolves from disorder to structures and their structures grow all under the gravitational interactions [96]. This should be entropy decrease. And the simulation must be an isolated system, because no matter can be added. They are systems bound by their own gravity, i.e., only using internal gravitational interactions.

Moreover, famous economist Schelling described a model with two red and white agents, which evolved from the initial high mixing to separate from each other $[97,98]$. This is similar to the separation of water-oil in a liquid or of metal alloys, and is just opposite to the process usual as a typical example of entropy increase. The corresponding magnetization and the orienting fields can produce similar effects. The interactions are stronger in social science, which will be more deviation from the uniformity. The various relations and feedback within the system are internal interactions. Interconnection is a typical and clear feature of modern society [98]. This also leads to the complexity of the entropy changes.

In a word, coherence, entanglement and astrophysical simulation models are shown the possibility of entropy decrease in some isolated systems.

\section{References}

1. Eddington AE (1928) The Nature of the Physical World. New York: Macmillan, pp: 74.

2. Bennett CH (1982) The thermodynamics of computation: a review. Iut J Theor Phys 21: 905-940.

3. Leff HS, Rex AF (1990) Maxwell's Demon: Entropy, Information, Computing. Taylor \& Francis, CRC Press, pp: 502.

4. Chang YF (1997) Possible decrease of entropy due to internal interactions in isolated systems. Apeiron 4: 9799.

5. Chang YF (2005) Entropy, fluctuation magnified and internal interactions. Entropy 7(3): 190-198.

6. Chang YF (2012) "Negative temperature" fallacy, sufficient-necessary condition on entropy decrease in isolated systems and some possible tests in physics, chemistry and biology. International Review of Physics 6: 469-475.

7. Chang YF (2013) Unified quantum statistics, possible violation of Pauli exclusion principle, nonlinear equations and some basic problems of entropy. International Review of Physics 7(4): 299-306.

8. Chang YF (2015) Entropy decrease in isolated system and its quantitative calculations in thermodynamics of microstructure. International Journal of Modern Theoretical Physics 4(1): 1-15.

9. Chang YF (2019) Self-organization, critical phenomena, entropy decrease in isolated systems and its tests. International Journal of Modern Theoretical Physics 8: 17-32.

10. Chang YF (2013) Chemical reactions and possible entropy decrease in isolated system. International Journal of Modern Chemistry 4(3): 126-136.

11. Chang YF (2014) Catalyst theory, entropy decrease in isolated system and transformation of internal energy. International Journal of Modern Chemistry 6(2): 74-86.

12. Chang YF (2013) Possible entropy decrease in biology and some new research of biothermodynamics. NeuroQuantology 11: 189-196.

13. Chang YF (2016) Bioinformation and twistor theory in biology. NeuroQuantology 14: 589-596.

14. Chang YF (2018) Entropy change in biological thermodynamics. International Journal of Research Studies in Biosciences 6(6): 5-12.

15. Chang YF (2013) Grand unified theory applied to gravitational collapse, entropy decrease in astronomy, singularity and quantum fluctuation. International Journal of Modern Applied Physics 3(1): 8-25. 
16. Chang YF (2018) Belief of entropy increase, fallacy of black hole thermodynamics, and its development. International Journal of Modern Applied Physics 8(1): 1-10.

17. Chang YF (2013) Social thermodynamics, social hydrodynamics and some mathematical applications in social sciences. International Journal of Modern Social Science 2(2): 94-108.

18. Chang YF (2015) Entropy economics, entropy sociology and some social developed pattern. International Journal of Modern Social Science 4(1): 42-56.

19. Chang YF (2020) Development of entropy change in philosophy of science. Philosophy Study 10(9): 517524.

20. Chang YF (2020) Entropy decrease in isolated systems: theory, fact and tests. International Journal of Fundamental Physical Sciences 10(2):16-25.

21. Wang GM, Sevick EM, Mittag E, Searles DJ, Evans DJ (2002) Experimental demonstration of violations of the second law of thermodynamics for small systems and short time scales. Phys Rev Lett 89(5): 050601.

22. Carberry DM, Reid JC, Wang GM, Sevick EM, Searles DJ, et al. (2004) Fluctuations and irreversibility: An experimental demonstration of a second-law-like theorem using a colloidal particle held in an optical trap. Phys Rev Lett 92(14): 140601.

23. Bouwmeester D, Pan JW, Mattle K, Eibl M, Weinfurter $\mathrm{H}$, et al. (1997) Experimental quantum teleportation. Nature 390: 575-579.

24. Zbiden H, Brendel J, Gisin N, Tittel W (2001) Experimental test of nonlocal quantum correlation in relativistic configurations. Phys Rev A 63: 022111.

25. Stapp HP (1979) Whiteheadian approach to quantum theory and the generalized Bell's theorem. Found Phys 9: 1-25.

26. Badurek G, Rauch H, Tuppinger D (1986) Neutron interferometric double-resonance experiment. Phys Rev A 34(4): 2600-2608.

27. Scully MO, Englert BG, Walther H (1991) Quantum optical tests of complementarity. Nature 351: 111-116.

28. Buks E, Schuster R, Heiblum M, Mahalu D, Umansky V (1998) Dephasing in electron interference by a whichpath detector. Nature 391: 871-874.

29. Durr S, Nonn T, Rampe G (1998) Origin of quantum mechanical complementary probed by a 'Which-Way' experiment in an atom interferometer. Nature 395: 3337.

30. Wheeler JA, Zurk WH (Ed.), (1983) Quantum Measurement. Princeton Univ. Press.

31. Monroe C, Meekhof DM, King BE, Wineland DJ (1996) A 'Schrodinger Cat' superposition state of an atom. Science 272(5265): 1131-1136.

32. Julsgaard B, Kozhekin A, Polzik ES (2001) Experimental long-lived entanglement of two macroscopic objects. Nature 413: 400-403.

33. Chang YF (1984) High energy behaviour of particles and unified statistics. Hadronic J 7: 1118-1133.

34. Chang YF (1984) Some possible tests of the inapplicability of Pauli's exclusion principle. Hadronic J 7: 1469-1473.

35. Chang YF (1992) Various theories relevant to possible violation of the Pauli principle. In: Myung HC (Ed.), Hadronic Mechanics and Nonpotential Interactions. Nova Science Publishers, Inc. pp: 169-175.

36. Chang YF (1999) Test of Pauli's exclusion principle in particle physics, astrophysics and other fields. Hadronic J 22: 257-268.

37. Chang YF (2020) At ultra-low energy possible violation of Pauli exclusion principle and its possible mechanism and predictions. Hadronic J 43: 161-169.

38. Chang YF (1990) New Research of Particle Physics and Relativity (Yunnan Science and Technology Press 1989). Phys Abst 93: 1371.

39. Chang YF (2013) Extension and complete structure of the special relativity included superluminal and neutrino-photon with mass. International Journal of Modern Theoretical Physics 2(2): 53-73.

40. Wang LJ, Kuzmich A, Dogariu A (2000) Gain-assisted superluminal light propagation. Nature 406: 277-279.

41. Mugnai D, Ramfagni A, Ruggeri R (2000) Observation of superluminal behavior in wave propagation. Phys Rev Lett 84(21): 4830-4833.

42. Marangos J (2000) Faster than a speeding photon. Nature 406: 243-244.

43. Zhang YD (2015) Advanced Quantum Mechanics. $3^{\text {rd }}$ (Edn.), Science Press, China.

44. Landau LD, Lifshitz EM (1980) Statistical Physics. Vol 9, 
Pergamon Press, UK.

45. Reichl LE (1980) A Modern Course in Statistical Physics. University of Texas Press, USA.

46. Nielsen MA, Chuang IL (2000) Quantum Computation and Quantum Information. Cambridge University Press, USA, pp: 1-704.

47. Vedral V (2006) Introduction to Quantum Information Science. Oxford University Press, USA.

48. Wilde MM (2017) Quantum Information Theory. $2^{\text {nd }}$ (Edn.), Cambridge University Press, USA.

49. Watrous J (2018) The Theory of Quantum Information. Cambridge University Press, USA.

50. Chitambar E, Hsieh MH (2016) Relating the Resource Theories of Entanglement and Quantum Coherence. Phys Rev Lett 117(2): 020402.

51. Baumgratz T, Cramer M, Plenio MB (2014) Quantifying coherence. Phys Rev Lett 113(14): 140401.

52. Streltsov A, Singh U, Dhar HS, Bera MN, Adesso G (2015) Measuring Quantum Coherence with Entanglement. Phys Rev Lett 115(2): 020403.

53. Du SP, Bai ZF, Guo Y (2015) Conditions for coherence transformations under incoherent operations. Phys Rev A 91(5): 052120.

54. Mani A, Karimipour A (2015) Cohering and decohering power of quantum channels. Phys Rev A 92(3): 032331.

55. Lostaglio M, Korzekwa K, Jennings D, Rudolph T (2015) Quantum Coherence, Time-Translation Symmetry, and Thermodynamics. Phys Rev X 5: 021001.

56. Ma J, Yadin B, Girolami D, Vedral V, Gu M (2016) Converting Coherence to Quantum Correlations. Phys Rev Lett 116: 160407.

57. Streltsov A, Chitambar E, Rana S, Bera MN, Winter A, et al. (2016) Entanglement and Coherence in Quantum State Merging. Phys Rev Lett 116(24): 240405.

58. Rana S, Parashar P, Lewenstein M (2016) Trace-distance measure of coherence. Phys Rev A 93(1): 012110.

59. Gärttner M, Hauke P, Rey AM (2018) Relating Out-ofTime-Order Correlations to Entanglement via MultipleQuantum Coherences. Phys Rev Lett 120(4): 040402.

60. Xi ZJ (2019) Entropy Measure of Non-classical Correlation and Quantum Coherence. Science Press, China.
61. Dry CM (1993) Passive smart materials for sensing and actuation. J Intelligent Materials Systems and Structures 4(3): 420-425.

62. Amato I (1992) Animating the material world. Science 255(5042): 284-286.

63. Langton CG (1984) Self-reproduction in cellular automata. Physica D 10: 135-144.

64. Langton CG (1989) Artificial Life. Addison Wesley, pp: 40-94.

65. Wolfram S (2002) A New Kind of Science. Wolfram Media, Inc.

66. Vicsek T, Czirok A, Ben-Jacob E, Cohen I, Shochet IO, et al. (1995) Novel type of phase transition in a system of self-driven particle. Phys Rev Lett 75(6): 1226-1229.

67. Toner J, Tu Y (1995) Long-rang order in a twodimensional dynamical XY model: How birds fly together. Phys Rev Lett 75(23): 4326-4329.

68. Ben-Jacob E, Cohen I, Levine H (2000) Cooperative selforganization of microorganisms. Adv Phys 49(4): 395554.

69. Scully MO, Zubairy MS, Agarwal GS, Walther H (2003) Extracting Work from a Single Heat Bath via Vanishing Quantum Coherence. Science 299(5608): 862-864.

70. Quan HT, Wang YD, Liu YX, Sun CP, Nori F (2006) Maxwell's Demon Assisted Thermodynamic Cycle in Superconducting Quantum Circuits. Phys Rev Lett 97(18):180402.

71. Hackermuler L, Hornberger K, Brezger B, Zeilinger A, Arndt M (2004) Decoherence of matter waves by thermal emission of radiation. Nature 427: 711-714.

72. Dodelson S (2003) Modern Cosmology. $1^{\text {st }}$ (Edn.), Academic Press, New York, USA.

73. Lemson G, Consortium V (2006) Halo and Galaxy Formation Histories from the Millennium Simulation: Public release of a VO-oriented and SQL-queryable database for studying the evolution of galaxies in the LambdaCDM cosmogony.

74. Harker G, Cole S, Helly J, Frenk C, Jenkins A (2006) A marked correlation function analysis of halo formation times in the Millennium Simulation. Mon Not R Astron Soc 367(3): 1039-1049.

75. Bett P, Eke V, Frenk CS, Jenkins A, Helly J, et al. (2007) The spin and shape of dark matter haloes in the Millennium 
simulation of a $\Lambda$ cold dark matter universe. Mon Not $\mathrm{R}$ Astron Soc 376(1): 215-232.

76. Michael BK, Volker S, White SDM, Adrian J, Gerard L (2009) Resolving Cosmic Structure Formation with the Millennium-II Simulation. Mon Not R Astron Soc 398(3):1150-1164.

77. Vogelsberger M, Genel S, Sijacki D, Torrey P, Springel V, et al. (2013) A model for cosmological simulations of galaxy formation physics. Mon Not R Astron Soc Soc 436(4): 3031-3067.

78. Vogelsberger M, Genel S, Springel V, Torrey P, Sijacki D, et al. (2014) Properties of galaxies reproduced by a hydrodynamic simulation. Nature 509: 177-182.

79. Gibney E (2014) Model universe recreates evolution of the cosmos. Nature 509: 177-182.

80. Brook CB, Stinson G, Gibson BK, Wadsley J, Quinn $\mathrm{T}$ (2012) MaGICC discs: matching observed galaxy relationships over a wide stellar mass range. Mon Not R Astron Soc 424(2): 1275-1283.

81. Quilis V, Trujillo I (2012) Satellites around massive galaxies since $\mathrm{z} \sim 2$ : confronting the millennium simulation with observations. Astrophys J 752: L19.

82. Scannapieco C, Wadepuhl M, Parry OH, Navarro JF, Jenkins A, et al. (2012) The Aquila comparison project: the effects of feedback and numerical methods on simulations of galaxy formation. Mon Not R Astron Soc 423(2): 1726-1749.

83. Springel V, Hernquist L (2003) Cosmological smoothed particle hydrodynamics simulations: a hybrid multiphase model for star formation. Mon Not R Astron Soc 339(2): 289-311.

84. Saro A, Borgani S, Tornatore L, Dolag K, Murante G, et al. (2006) Properties of the galaxy population in hydrodynamical simulations of clusters. Mon Not $\mathrm{R}$ Astron Soc 373(1): 397-410.

85. Libeskind NI, Cole S, Frenk CS, Okamoto T, Jenkins A (2007) Satellite systems around galaxies in hydrodynamic simulations. Mon Not R Astron Soc 374(1): 16-28.
86. Sijacki D, Springel V, Di Matteo T, Hernquist L (2007) A unified model for AGN feedback in cosmological simulations of structure formation. Mon Not R Astron Soc 380(3): 877-900.

87. Dolag K, Borgani S, Murante G, Springel V (2009) Substructures in hydrodynamical cluster simulations. Mon Not R Astron Soc 399(2): 497-514.

88. Springel V (2010) E pur si muove: Galilean-invariant cosmological hydrodynamical simulations on a moving mesh. Mon Not R Astron Soc 401(2): 791-851.

89. Wiersma RPC, Schaye J, Theuns T (2011) The effect of variations in the input physics on the cosmic distribution of metals predicted by simulations. Mon Not R Astron Soc 415(1): 353-371.

90. Genel S (2013) Following the flow: tracer particles in astrophysical fluid simulations. Mon Not R Astron Soc 435: 1426-1442.

91. Marinacci F, Pakmor R, Springel V (2014) The formation of disc galaxies in high-resolution movingmesh cosmological simulations. Mon Not R Astron Soc 437(2): 1750-1775.

92. Lang KR (1974) Astrophysical Formulae. $2^{\text {nd }}(E d n$.$) ,$ Springer-Verlag Berlin Heidelberg, pp: 786.

93. Harwit M (1973) Astrophysical Concepts. $2^{\text {nd }}(E d n$.$) ,$ John Wiley and Sons, pp: 626.

94. Rifkin J, Toward T (1981) Entropy, a New World View. Bantam Edition, New York, Toronto.

95. Hawking S (1974) Black hole evaporation. Nature 248: 30-31.

96. Mazure A, Le Brun V (2011) Matter, Dark Matter, and Anti-Matter. Springer-Verlag, New York Inc.

97. Schelling TC (1978) Micromotives and Macrobehavior. WW Norton, New York.

98. Ball P (2012) Why Society Is a Complex Matter: Meeting Twenty-first Century Challenges with a New Kind of Science. $1^{\text {st }}$ (Edn.), Springer-Verlag Berlin Heidelberg, pp: 60. 